\title{
Diabetes impairs the angiogenic potential of adipose-derived stem cells by selectively depleting cellular subpopulations
}

\author{
Robert C Rennert ${ }^{1 \dagger}$, Michael Sorkin $^{1 \dagger}$, Michael Januszyk ${ }^{1}$, Dominik Duscher ${ }^{1}$, Revanth Kosaraju ${ }^{1}$, Michael T Chung ${ }^{1}$, \\ James Lennon', Anika Radiya-Dixit', Shubha Raghvendra', Zeshaan N Maan', Michael S Hu', Jayakumar Rajadas², \\ Melanie Rodrigues ${ }^{1}$ and Geoffrey C Gurtner ${ }^{1 *}$
}

\begin{abstract}
Introduction: Pathophysiologic changes associated with diabetes impair new blood vessel formation and wound healing. Mesenchymal stem cells derived from adipose tissue (ASCs) have been used clinically to promote healing, although it remains unclear whether diabetes impairs their functional and therapeutic capacity.

Methods: In this study, we examined the impact of diabetes on the murine ASC niche as well as on the potential of isolated cells to promote neovascularization in vitro and in vivo. A novel single-cell analytical approach was used to interrogate ASC heterogeneity and subpopulation dynamics in this pathologic setting.

Results: Our results demonstrate that diabetes alters the ASC niche in situ and that diabetic ASCs are compromised in their ability to establish a vascular network both in vitro and in vivo. Moreover, these diabetic cells were ineffective in promoting soft tissue neovascularization and wound healing. Single-cell transcriptional analysis identified a subpopulation of cells which was diminished in both type 1 and type 2 models of diabetes. These cells were characterized by the high expression of genes known to be important for new blood vessel growth.
\end{abstract}

Conclusions: Perturbations in specific cellular subpopulations, visible only on a single-cell level, represent a previously unreported mechanism for the dysfunction of diabetic ASCs. These data suggest that the utility of autologous ASCs for cell-based therapies in patients with diabetes may be limited and that interventions to improve cell function before application are warranted.

\section{Introduction}

Diabetes mellitus (DM) is associated with significant impairments in neovascularization and wound healing [1]. Although the exact mechanism underlying this pathology remains unknown, there is evidence for diabetesassociated dysfunction at both the cellular and molecular level. Specifically, diabetes has been linked to impairments in the functionality of diabetic endothelial progenitor cells (EPCs) and resident tissue fibroblast in vitro $[2,3]$. Additionally, reduced local expression of the vasculogenic and regenerative cytokines vascular endothelial

\footnotetext{
* Correspondence: ggurtner@stanford.edu

${ }^{\dagger}$ Equal contributors

'Hagey Laboratory for Pediatric Regenerative Medicine; Department of Surgery, Stanford University School of Medicine, 257 Campus Drive West, Hagey Building GK-201, Stanford, CA 94305-5148, USA

Full list of author information is available at the end of the article
}

cell growth factor (VEGF) and hypoxia-inducible factor 1-alpha (HIF-1 $\alpha)$ has been reported in diabetic tissues following injury $[4,5]$.

A variety of treatment approaches that seek to address the specific deficiencies present in diabetic wounds have been developed. Cell-based therapies, in particular, represent an appealing treatment paradigm, as they potentially contribute both cytokines and a cellular framework to the tissue regeneration process. In support of this approach, multiple cell-based products delivering fibroblasts or fibroblast-keratinocyte mixtures have a proven clinical efficacy for the treatment of diabetic wounds [6]. Moreover, advanced biomaterials are being developed to optimize cell survival and functionality within the harsh wound environment [7-9].

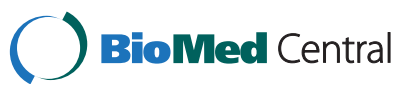


Adipose-derived mesenchymal stem cells (ASCs) are an especially appealing therapeutic cell source because of their multipotency, paracrine secretion of growth factors and cytokines [10], and demonstrated efficacy in models of wound healing and ischemic revascularization $[11,12]$. Additionally, adipose tissue presents a reservoir of readily available cells, which can be easily harvested and directly employed for autologous clinical application [13]. This methodology obviates the need for ex vivo cell expansion while avoiding the immunogenic concerns associated with allogeneic cell transplantation. The value of autologous cell therapies in patients with diabetes may be limited, however, as stem cell phenotypes can be negatively impacted by diabetes [14,15], and diabetic ASCs display an impaired neovascular potential in vitro [16]. Nonetheless, the therapeutic efficacy of diabetic ASCs within cutaneous wounds remains unclear [17].

Single-cell analytical approaches facilitate the in-depth exploration of cell heterogeneity and subpopulation dynamics, and we have recently used this technique to characterize stem cell phenotypes [18] and enhance ASC functionality via predictive subpopulation enrichment [19]. Importantly, determining the effect of diabetes on ASCs with single-cell granularity is the only way to differentiate between a global effect on cell functionality and a more selective subpopulation-level influence. This information is critically important for the design of effective autologous ASC-based therapies in patients with diabetes, as a global cell dysfunction would demand ex vivo modulation prior to application, whereas subpopulationlevel effects may be overcome through more selective cell enrichment. In this study, we examine the impact of diabetes on the ASC niche as well as the ability of ASCs to promote neovascularization and wound healing when delivered within a biomimetic hydrogel scaffold developed in our laboratory $[7,8]$. Finally, we interrogate these cells on a single-cell level to characterize ASC population dynamics associated with this pathologic state.

\section{Methods}

\section{Animals}

Wild-type (WT) (C57BL/6) and type 2 diabetic (DM2) mice (BKS.Cg-m $+/+$ Lepr $^{d b}$ ) were purchased from Jackson Laboratories (Bar Harbor, ME, USA) to study the effect of diabetes on ASC physiology. Selected assays were also performed on ASCs isolated from WT mice following induction of type 1 diabetes (DM1) via streptozotocin injection (STZ) (Sigma-Aldrich, St. Louis, MO, USA) as previously described [20]. Only mice with blood glucose levels of greater than $350 \mathrm{mg} / \mathrm{dL}$ were considered diabetic and used for further analysis. All protocols were approved by the Stanford Administrative Panel on Laboratory Animal Care.

\section{ASC niche analysis}

WT and DM2 murine inguinal fat pads were harvested and manually disrupted for real-time quantitative polymerase chain reaction as described below.

\section{ASC harvest and culture}

ASCs were isolated from WT, DM1, and DM2 murine inguinal fat pads, minced, and digested for 1 hour at $37^{\circ} \mathrm{C}$ using collagenase I (Roche Applied Science, Indianapolis, IN, USA). The reaction was stopped with the addition of supplemented media, pelleted via centrifugation, and subjected to erythrocyte lysis in accordance with the instructions of the manufacturer (Sigma-Aldrich). The remaining cells were pelleted again, forming the stromal vascular fraction (SVF). The SVF was either cultured under standard conditions $\left(37^{\circ} \mathrm{C}\right.$ in $5 \% \mathrm{CO}_{2}$ ) in Dulbecco's modified Eagle's medium (DMEM) with 10\% fetal bovine serum (FBS) and 1\% penicillin/streptomycin (Life Technologies, Grand Island, NY, USA) containing $1 \mathrm{~g} / \mathrm{L}$ of glucose to purify for ASCs or used immediately for flow cytometric/ single-cell analyses. Cultured cells were used at or before passage 2, and all analyses were run in triplicate unless otherwise stated.

\section{In vitro Matrigel tubulization assays}

PKH26-labeled WT and DM2 ASCs alone or mixed with calcein-labeled human umbilical vein endothelial cells (HUVECs) (Life Technologies) were cultured for 12 hours under hypoxic conditions on a 24 -well plate $\left(4 \times 10^{4}\right.$ cells per well) coated with growth factor-reduced Matrigel (BD Biosciences, Franklin Lakes, NJ, USA). ASC and HUVEC tubule counts were determined in five random high-power fields per well, respectively, by using an inverted Leica DMIL microscope (Leica Microsystems, Wetzlar, Germany).

\section{In vivo Matrigel plug assay and CD31 immunohistochemistry}

WT or DM2 ASCs $\left(8 \times 10^{5}\right)$ (cultured not more than two passages) were suspended in $250 \mu \mathrm{L}$ of growth factorreduced Matrigel (BD Biosciences) and injected in a subcutaneous fashion on the dorsum of WT mice $(n=4)$. Plugs were harvested at day 10 , and $7-\mu \mathrm{m}$-thick frozen sections were immunohistochemically stained for the commonly used vascular marker platelet/endothelial cell adhesion molecule 1 (PECAM1/CD31, a transmembrane glycoprotein expressed on the surface of platelets, endothelial cells, and subsets of hematopoietic cells but particularly concentrated at the intercellular junctions of endothelial cells), followed by ImageJ (National Institutes of Health, Bethesda, MD, USA) quantification [7,21]. 


\section{In vitro adipogenic differentiation}

WT and DM2 ASCs were seeded in standard six-well tissue culture plates $\left(1.5 \times 10^{5}\right.$ cells per well $)$, and adipogenic differentiation medium-consisting of DMEM ( $1 \mathrm{~g} / \mathrm{L}$ glucose), $10 \%$ fetal bovine serum, $1 \%$ penicillin/streptomycin, $10 \mu \mathrm{g} / \mathrm{mL}$ insulin, $1 \mu \mathrm{M}$ dexamethasone, $0.5 \mathrm{mM}$ methylxanthine, and $200 \mu \mathrm{M}$ indomethacin-was added after cell attachment. Oil red $\mathrm{O}$ staining was performed after 7 days of incubation.

\section{In vitro osteogenic differentiation}

WT and DM2 ASCs were seeded in standard six-well tissue culture plates $\left(1.0 \times 10^{5}\right.$ cells per well $)$ and grown to at least $80 \%$ confluence before being cultured in osteogenic differentiation medium, which consisted of DMEM (1 g/L glucose) supplemented with 10\% FBS, 1\% penicillin/streptomycin, $100 \mu \mathrm{g} / \mathrm{mL}$ ascorbic acid, and $10 \mathrm{mM} \beta$-glycerophosphate. Photometric quantification of Alizarin red stain was performed after 14 days to assay extracellular mineralization as previously described [22].

\section{In vitro hydrogel bioscaffold seeding}

WT and DM2 ASCs $\left(1 \times 10^{5}\right)$ were suspended in $15 \mu \mathrm{L}$ of growth media and seeded within a previously described $5 \%$ collagen-pullulan hydrogel bioscaffold $[7,8]$. Seeded scaffolds were placed in growth media and incubated at $37^{\circ} \mathrm{C}$ in $5 \% \mathrm{CO}_{2}$ prior to proliferation and survival analyses and RNA/protein harvest.

\section{In vitro proliferation and survival}

After hydrogel bioscaffold seeding, a live-dead assay (Live/Dead Cell Viability Assay) was performed at multiple time points to assess WT and DM2 ASC viability in accordance with the instructions of the manufacturer (Life Technologies). ASC proliferation was compared between hydrogel-seeded WT and DM2 cells at multiple time points by using an MTT assay (Vybrant MTT Cell Proliferation Assay Kit; Invitrogen, Grand Island, NY, USA).

\section{Real-time quantitative polymerase chain reaction}

Total RNA was isolated from ground WT and DM2 fat pads or hydrogel-seeded ASCs by using an RNeasy Mini Kit (Qiagen, Germantown, MD, USA) and transcribed to cDNA (Superscript First-Strand Synthesis Kit; Invitrogen). Real-time quantitative polymerase chain reactions (qPCRs) were performed by using TaqMan gene expression assays (Applied Biosystems, Foster City, CA, USA) for murine $\mathrm{Mmp}$-9 (matrix metalloproteinase 9, Mm00442991_m1), Cxcl-12 (stromal cell-derived factor1/Sdf-1, Mm00445552_m1), Vegf-a (vascular endothelial growth factor-A, Mm01281447_m1), Eng (endoglin, Mm00468256_m1), Hgf (hepatocyte growth factor, Mm01135193_m1), Mmp-3 (matrix metalloproteinase 3,
Mm00440295_m1), Cxcr-4 (chemokine receptor 4, Mm01292123_m1), Fgf-2 (fibroblast growth factor 2, Mm00433287_m1), Fgfr-2 (fibroblast growth factor receptor 2, Mm01269930_m1), Pdgf-a (platelet-derived growth factor-A, Mm01205760_m1), Pdfgr-a (plateletderived growth factor receptor-A, Mm01205760_m1), and Angpt-1 (angiopoietin 1, Mm00456503_m1) by using a Prism 7900HT Sequence Detection System (Applied Biosystems). Expression levels of the target genes were normalized to Actb (beta actin, Mm01205647_g1) or B2m (beta-2-microglobulin, Mm00437764_m1).

\section{Angiogenesis array}

Angiogenic cytokine protein production from hydrogelseeded WT and DM2 ASCs was quantified by using a Mouse Angiogenesis Array Kit (R\&D Systems, Minneapolis, MN, USA). Pixel density of each spot in the array was quantified and normalized to controls by using ImageJ (National Institutes of Health).

\section{In vivo murine ischemia model}

A model of graded soft tissue ischemia was created on the dorsum of WT mice, as described previously [23]. Briefly, a full-thickness three-sided peninsular flap was elevated, and a thin silicone sheet was inserted to separate the skin from the underlying fascia. A size-matched hydrogel bioscaffold was inlaid between the silicon sheet and skin flap either alone or following the seeding of $2.5 \times 10^{6}$ WT or diabetic (type 2 or 1 ) ASCs (cultured not more than two passages) $(\mathrm{n}=5)$. Unseeded scaffolds were used as controls. The skin flap was then sutured into place, and the demarcating necrotic area was quantified 10 days post-op. The proximal surviving flap was processed for neovascular growth via CD31 immunohistochemistry.

\section{Microfluidic single-cell gene expression analysis}

ASCs isolated from freshly harvested WT, DM2, and DM1 SVF (obtained as described above) by using the surface marker profile $\mathrm{CD} 45^{-} / \mathrm{CD} 31^{-} / \mathrm{CD} 34^{+}$(to exclude contaminating $\mathrm{CD}_{4} 5^{+}$hematopoietic and $\mathrm{CD} 31^{+}$endothelial cells found within the SVF and to select for a putatively stem-like subset of $\mathrm{CD}^{+} 4^{+}$cells $\left.[22,24]\right)$ were analyzed and sorted as single cells by using a Becton Dickinson FACSAria flow cytometer (Becton Dickinson, Franklin Lakes, NJ, USA) into $6 \mu \mathrm{L}$ of lysis buffer. Propodium iodide exclusion was used to ensure that only live cells were sorted. Reverse transcription and low cycle pre-amplification were performed by using Cells Direct (Invitrogen) with TaqMan assay primer sets (Applied Biosystems) in accordance with the specifications of the manufacturers. cDNA was loaded onto 96.96 Dynamic Arrays (Fluidigm, South San Francisco, CA, USA) for qPCR amplification by using Universal PCR Master Mix 
(Applied Biosystems) with a uniquely compiled TaqMan assay primer set as previously described [18].

\section{Statistical analysis}

Results are presented as mean \pm standard error of the mean. Data analysis was performed by using a Student $t$ test. Results were considered significant for $P$ values of not more than 0.05. For single-cell transcriptional data, a Kolmogorov-Smirnov (K-S) test was used to compare empirical distributions, followed by an adaptive fuzzy cmeans clustering algorithm as previously described [18].

\section{Results}

Diabetes negatively impacts the ASC niche and impairs ASC promotion of neovascularization

To determine the impact of diabetes on the ASC niche in situ, the transcriptional profiles of DM2 and WT murine fat pads were analyzed. Indicative of a dysfunctional signaling environment in situ, the expression of multiple growth factors and cytokines (Vegf-a, Fgf-2, Pdfg-a, and $S d f-1 ; P<0.02)$ as well their associated receptors $(C x c r-4$, Fgfr-2, and $P d g f r-a ; P<0.01)$ was significantly decreased in the setting of diabetes (Figure 1A).

\section{A)}

B) WT-ASCS

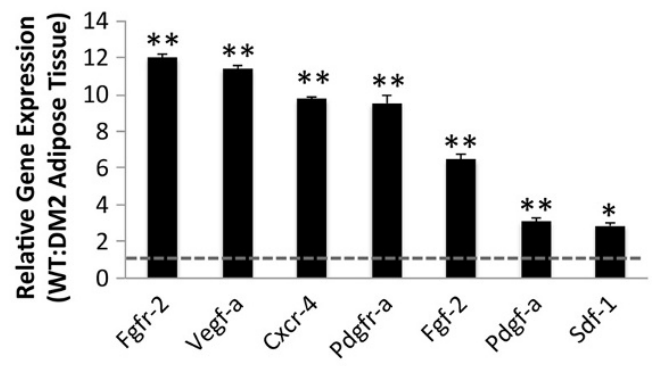

DM2-ASCS

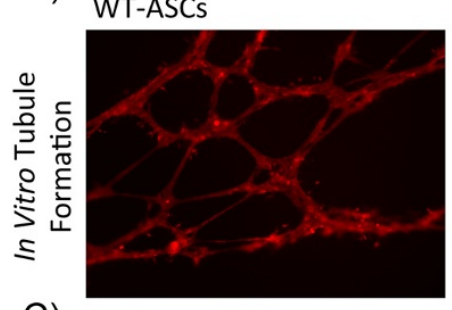

C)

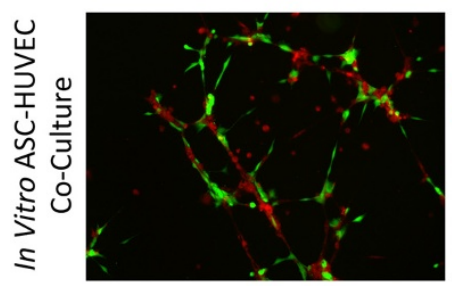

D)

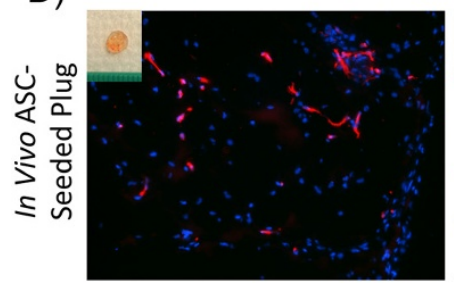

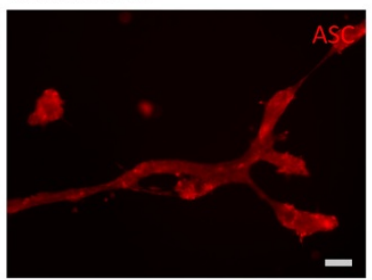
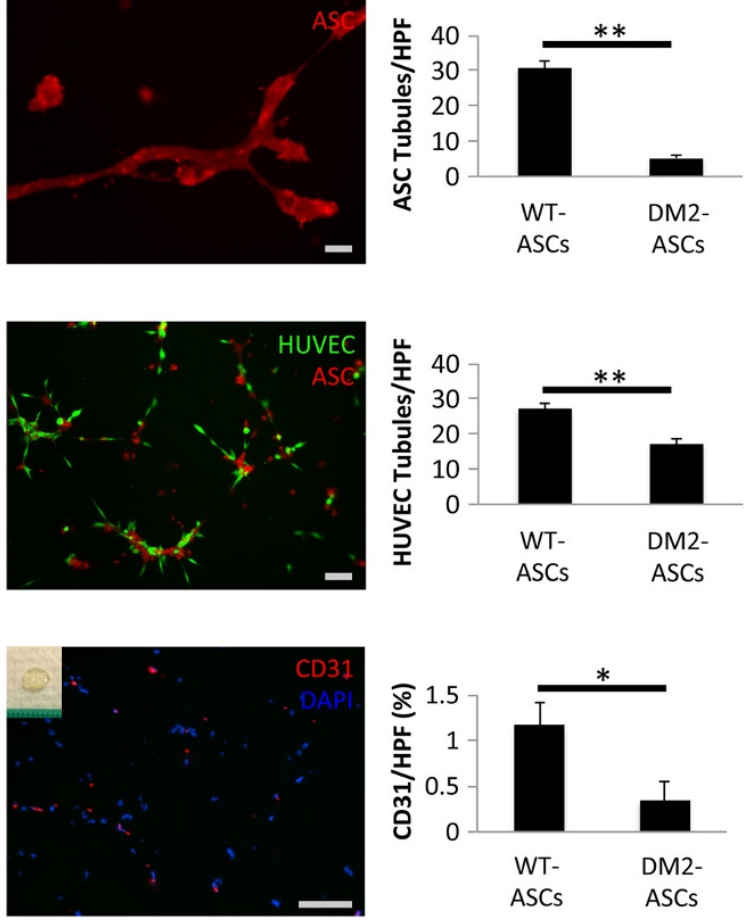

Figure 1 Adipose-derived mesenchymal stem cell (ASC) niche characterization and in vitro and in vivo analyses of ASC neovascular potential. (A) Transcriptional profile of type 2 diabetes mellitus (DM2) and wild-type (WT) murine fat pads. A dysfunctional in situ signaling environment was observed in the setting of diabetes. (B) Matrigel culture of WT and DM2 ASCs under hypoxic conditions. Tubules per high-power field (HPF) were quantified as a surrogate for direct ASC vasculogenic potential. (C) Matrigel co-culture of ASCs and human umbilical vein endothelial cells (HUVECS) under hypoxic conditions. HUVEC tubules per HPF were quantified as a measure of ASC-mediated endothelial network formation. (D) CD31 staining to quantify in vivo Matrigel plug vascularity following seeding with either WT or DM2 ASCs. Insets provide gross images of explanted plugs. Scale bar $=50 \mu \mathrm{m}$. ${ }^{*} P \leq 0.05$, ${ }^{*} P<0.01$. 
To next assess the potential of isolated diabetic ASCs to support the generation of vascular structures, we conducted a Matrigel tubule formation assay. When seeded alone, DM2 ASCs displayed significantly fewer tubular structures compared with WT cells (5.2 versus 30.6 tubules per HPF, respectively; $P<0.001$ ) (Figure $1 \mathrm{~B}$ ), suggesting a deficient response to extracellular matrix stimuli. ASCs are known to mediate angiogenesis mainly through paracrine mechanisms, however, as opposed to direct endothelial differentiation [25]. To analyze the ability of ASCs to promote endothelial sprouting, WT and DM2 ASCs were co-seeded with HUVEC cells by using the same model. Interestingly, although both ASC groups similarly localized around the HUVECs, consistent with their perivascular nature, the DM2 ASCs supported significantly less HUVEC tubule formation compared with WT ASCs (16.9 versus 27.1 tubules per HPF; $P=0.001$ ) (Figure $1 C$ ), indicating that diabetic ASCs possess a reduced stimulatory capacity.
To determine whether the observed in vitro findings translated into impaired in vivo neovascularization, we performed an assay in which Matrigel plugs seeded with DM2 or WT ASCs were implanted into subcutaneous pockets on the dorsum of WT mice, and new vessel formation was histologically assessed after 10 days (a time point at which neovascular processes are active following injury [26]). Consistent with our in vitro findings, plugs containing DM2 ASCs displayed significantly reduced levels of vascularization (as determined by CD31 immunohistochemistry) compared with plugs seeded with WT ASCs $(0.38$ versus $1.16 \%$ CD31 staining per HPF; $P=0.02$ ) (Figure 1D). Further exploring the effect of diabetes on ASC stemness, DM2 ASCs also displayed impairments in adipogenic and osteogenic differentiation in vitro (Figure 2), consistent with a loss of cell stemness and a phenotypic imprinting despite correction of the glucotic environment. Together, these data demonstrate that

A)

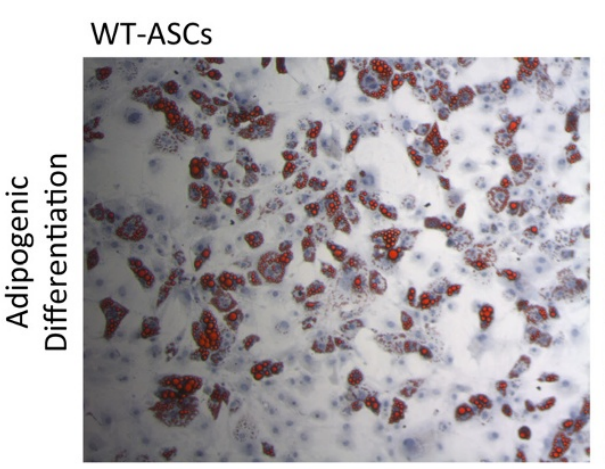

DM2-ASCs
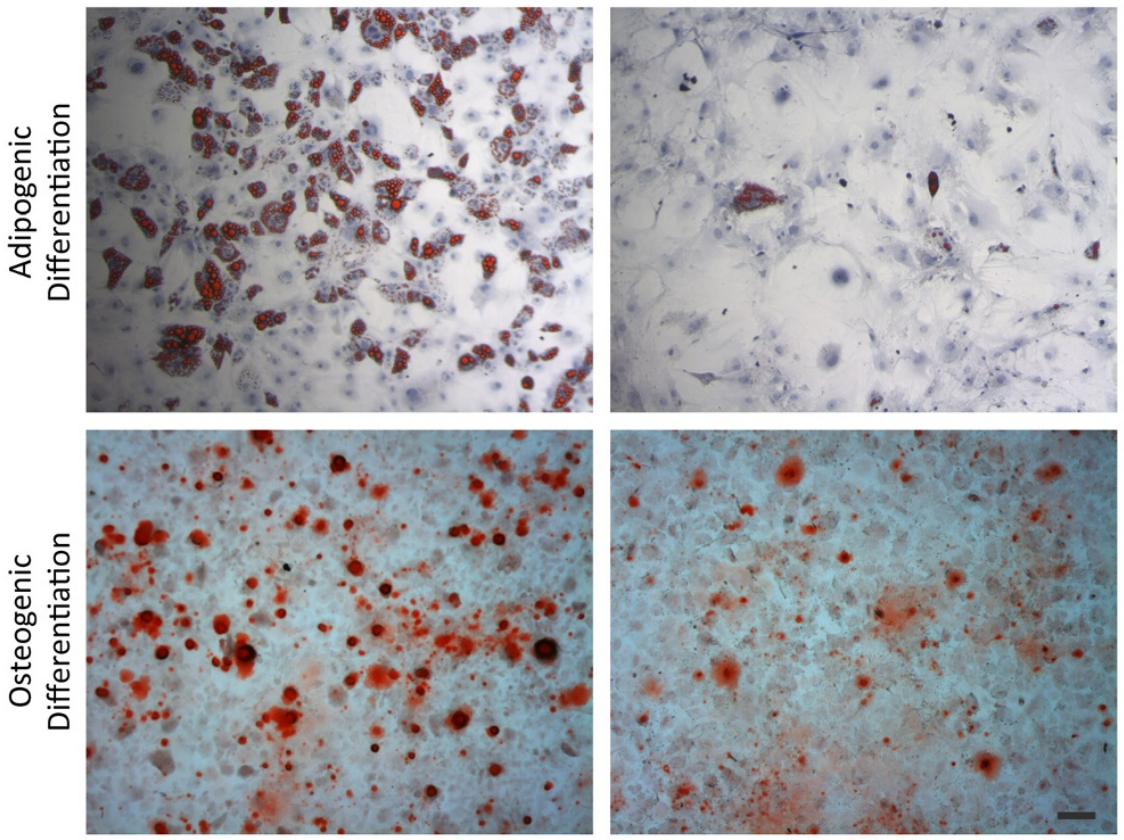

B)
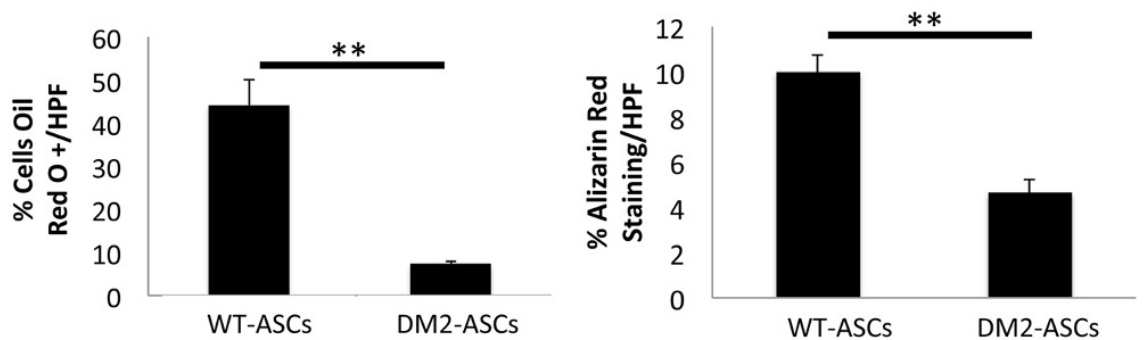

Figure 2 Adipose-derived mesenchymal stem cell (ASC) adipogenic and osteogenic differentiation. (A) Representative images and (B) quantification of Oil red $\mathrm{O}$ and Alizarin red staining following adipogenic and osteogenic differentiation of WT and DM2 ASCs. Scale bar $=50 \mu \mathrm{m}$. ${ }^{*} P<0.01$. 
diabetes significantly impairs the stemness and potential of ASCs to promote neovascularization both in vitro and in vivo.

\section{Optimization of ex vivo environment does not restore diabetic ASC function}

The delivery of cells to a wound site is particularly challenging because of a hostile environment characterized by inflammation and poor nutritional support. We have recently developed a biomimetic hydrogel scaffold, which has been shown to support MSC survival, promote 'stemlike' features, and improve pro-angiogenic functionality $[7,8]$. To further characterize the effect of diabetes on the angiogenic potential of ASCs and to determine whether this function can be restored, we examined the cellular response to seeding in this biomimetic hydrogel. Although WT and DM2 ASCs displayed a similar seeding efficiency and proliferation capacity within the hydrogel (Figure 3), diabetic ASCs possessed a markedly abnormal morphology (Figure 4A). Specifically, DM2 ASCs appeared to be rounded and clumped and failed to form the cytoplasmic extensions seen with WT cells, indicative of a lack of cell interaction with the scaffold microenvironment. Importantly, this phenotypic dysfunction in DM2 ASCs was accompanied by a decrease in transcriptional expression and production of several vasculogenesis-related and tissue remodeling mediators, including VEGF, MMP-9, and HGF $(P \leq 0.03)$ (Figure 4B,C).

\section{Diabetic ASCs fail to improve ischemic wound healing in vivo}

Diminished perfusion and neovascularization are important factors contributing to impaired diabetic healing; so to evaluate the regenerative potential of diabetic ASCs under representative conditions, cell-seeded biomimetic hydrogels were used in an adapted murine model of skin flap ischemia (Figure 5A,B) [27]. Although the application of WT ASCs showed a significant improvement in tissue survival at day 10 as compared with controls $(48.0 \%$ versus $22.3 \%$ flap survival; $P=0.02$ ), flaps treated with DM2 ASCs developed ischemic tissue necrosis at a level similar to that of the cell-free controls $(21.0 \%$ versus $22.3 \%$ flap survival; $P=0.87$ ) (Figure $5 \mathrm{C}$ ). This difference in tissue survival correlated with a deficiency of DM2 ASCs in promoting new blood vessel formation within the surviving proximal flap as compared to WT ASCs (1.12\% versus $2.34 \%$ CD31 staining per HPF; $P=0.04$ ) (Figure 5D,E). This time point is of particular relevance as it is within the window of physiologic neovascularization that occurs following injury but prior to vascular regression that occurs as part of the normal tissue remodeling process [26]. Moreover, these results were recapitulated with ASCs isolated from streptozotocininduced (DM1) mice, indicating that the observed findings were not model-specific, but rather reflect the impact of prolonged hyperglycemia on ASC physiology (Figure 5C-E). In aggregate, these data confirm the impaired vasculogenic potential of diabetic ASCs in vivo, despite delivery within a biomimetic scaffold.

\section{Diabetes selectively depletes a subpopulation of ASCs} with a highly vasculogenic transcriptional profile

Because cell culture and/or surface marker based enrichment of ASCs from the heterogeneous SVF is often employed prior to application, understanding the effect of diabetes on ASC population dynamics in the SVF, as well as following purification, is critical. To first determine the influence of diabetes on global ASC levels within the SVF, a flow cytometric analysis was performed on WT, DM2, and DM1 samples for the presence of putative ASCs (CD45 / $\mathrm{CD} 1^{-} / \mathrm{CD}^{+} 4^{+}$cells). Indicative of a detrimental effect of diabetes on the global ASC population, both the DM2 and DM1 SVF displayed a reduced frequency of putative ASCs when compared with WT samples $(P<0.01)$ (Figure 6).

Although this global ASC depletion provides an explanation for the dysfunction of diabetic SVF, a finer degree of granularity is needed to analyze purified ASCs for phenotypic differences, especially as there is evidence
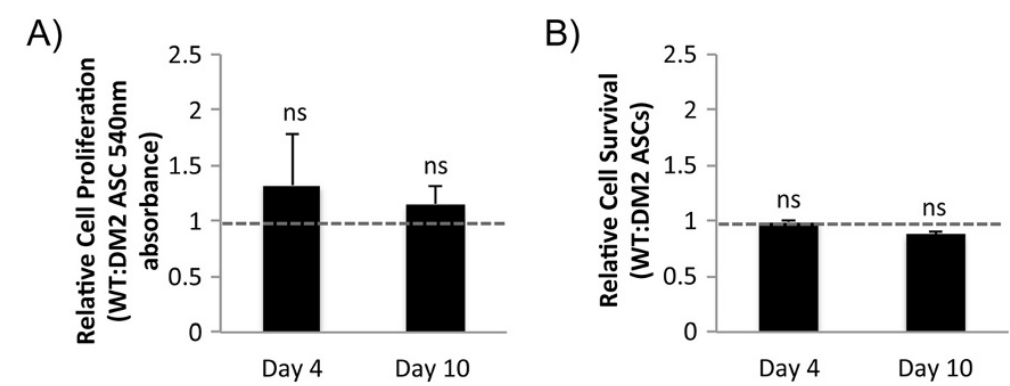

Figure 3 ASC behavior following in vitro hydrogel seeding. (A,B) Wild-type (WT) and diabetic (DM2) ASCs display similar rates of (A) proliferation and (B) survival in vitro following seeding within a three-dimensional bioscaffold. ns, not significant $(P>0.05)$. 


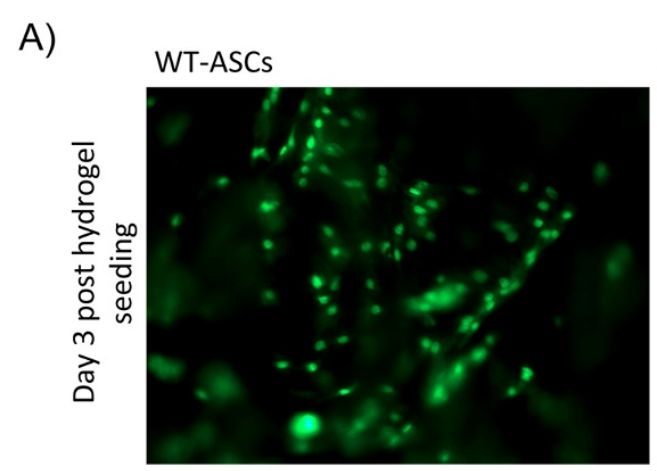

\section{DM2-ASCs}
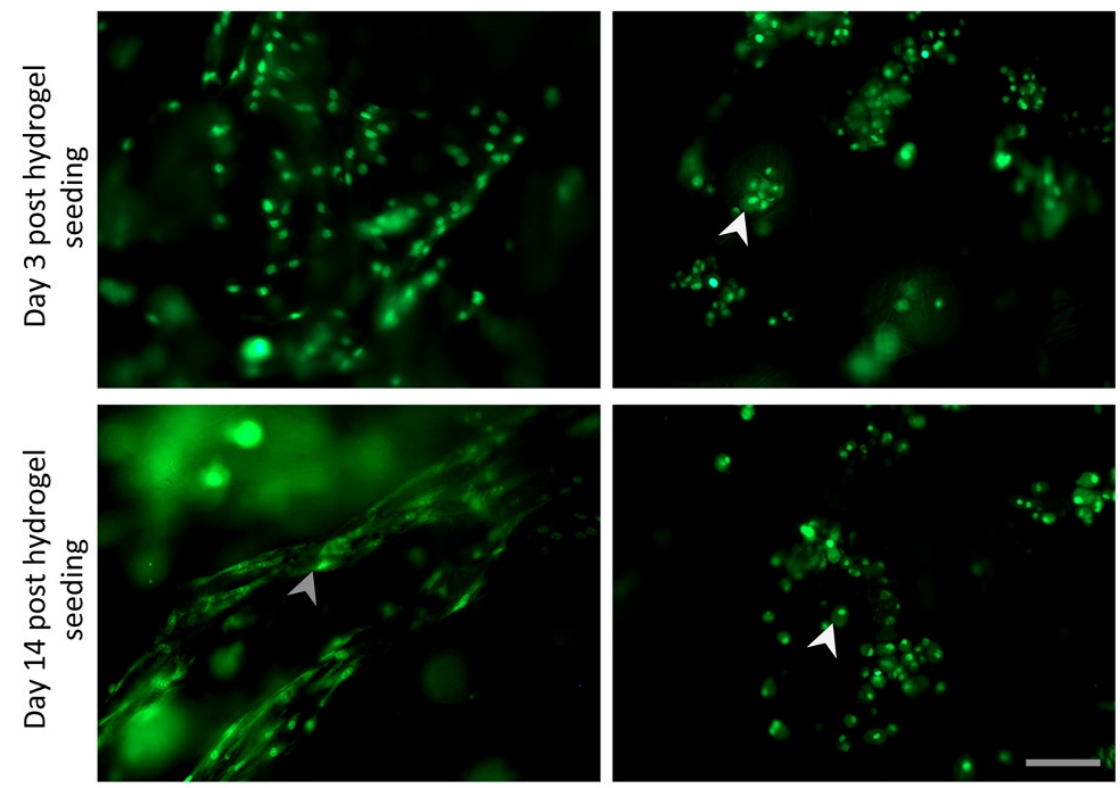

B)

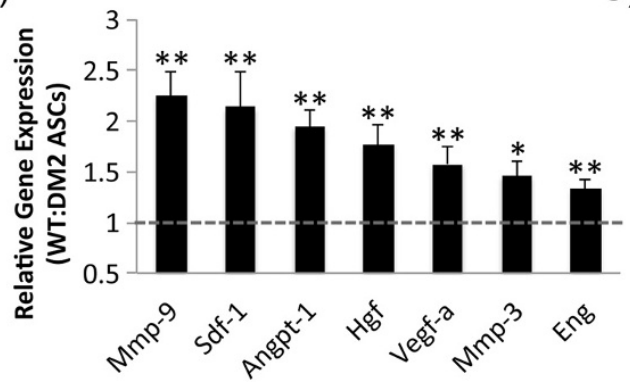

C)

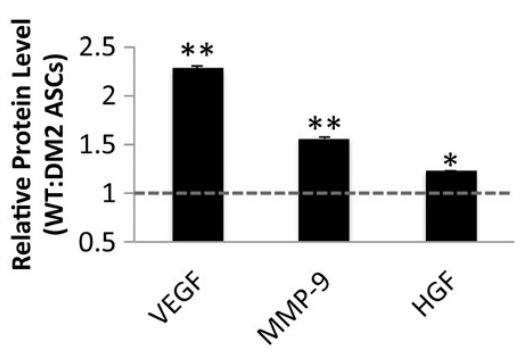

Figure 4 Adipose-derived mesenchymal stem cell (ASC) incorporation and secretory function following hydrogel bioscaffold seeding. (A) Wild-type (WT) and type 2 diabetes mellitus (DM2) ASCs at day 3 and 14 after hydrogel seeding. WT cells showed a uniform incorporation within the hydrogel and developed cell elongations and cytoplasmic extensions indicative of interactions with the bioscaffold (gray arrowhead). DM2 ASCs displayed a clumped distribution with rounded cell morphology (white arrowheads). (B) Reverse transcription-polymerase chain reaction and protein array $(\mathbf{C})$ quantifying the relative expression of selected genes associated with vasculogenenesis and tissue remodeling in hydrogel-seeded WT versus DM2 ASCs. Scale bar $=50 \mu \mathrm{m} .{ }^{*} P \leq 0.05,{ }^{* *} P<0.01$. Angpt-1, angiopoietin 1; Eng, endoglin; Hgf, hepatocyte growth factor; Mmp-3, matrix metalloproteinase 3; Mmp-9, matrix metalloproteinase 9; Sdf-1, Stromal Cell-Derived Factor 1; Vegf, vascular endothelial cell growth factor.

that even sorted ASCs consist of several subpopulations with varying levels of functionality [19]. To determine the effect of diabetes on ASC subpopulation dynamics, a single-cell interrogation of WT, DM2, and DM1 ASCs was conducted. For this, we employed microfluidic-based single-cell gene expression analysis as previously described [18]. Transcriptional profiles were simultaneously evaluated for approximately 70 gene targets across 75 individual cells per group, specifically looking at genes relating to stemness, vasculogenesis, and tissue regeneration (Additional file 1: Table S1). These cells were primary ASCs isolated based on a primitive surface marker definition $\left(\mathrm{CD} 45^{-} / \mathrm{CD} 31^{-} / \mathrm{CD} 34^{+}\right)$intended to exclude contaminating hematopoietic and endothelial cells found within the SVF.
When this approach was used, cells isolated from both WT and diabetic mice were found to display significant heterogeneity at the single-cell level (Figure 7A and Additional file 2: Figure S1). Moreover, KolmogorovSmirnov analysis of these single-cell data identified multiple genes that were differentially expressed in WT versus type 2 or 1 diabetic ASCs, including genes related to tissue remodeling, vasculogenesis, and cell differentiation, such as the metalloproteinases Mmp-3 and Adam-10, the chemokine $\mathrm{Ccl}-2$, and the transcription factors Hif-1a and $M e f-2 c(P<0.01)$ (Figure 7B) [28-32]. To determine whether these differences were the result of changes in specific subpopulations of diabetic or WT ASCs, partitional clustering was applied to the super-set of transcriptional profiles encompassing WT and diabetic cells 


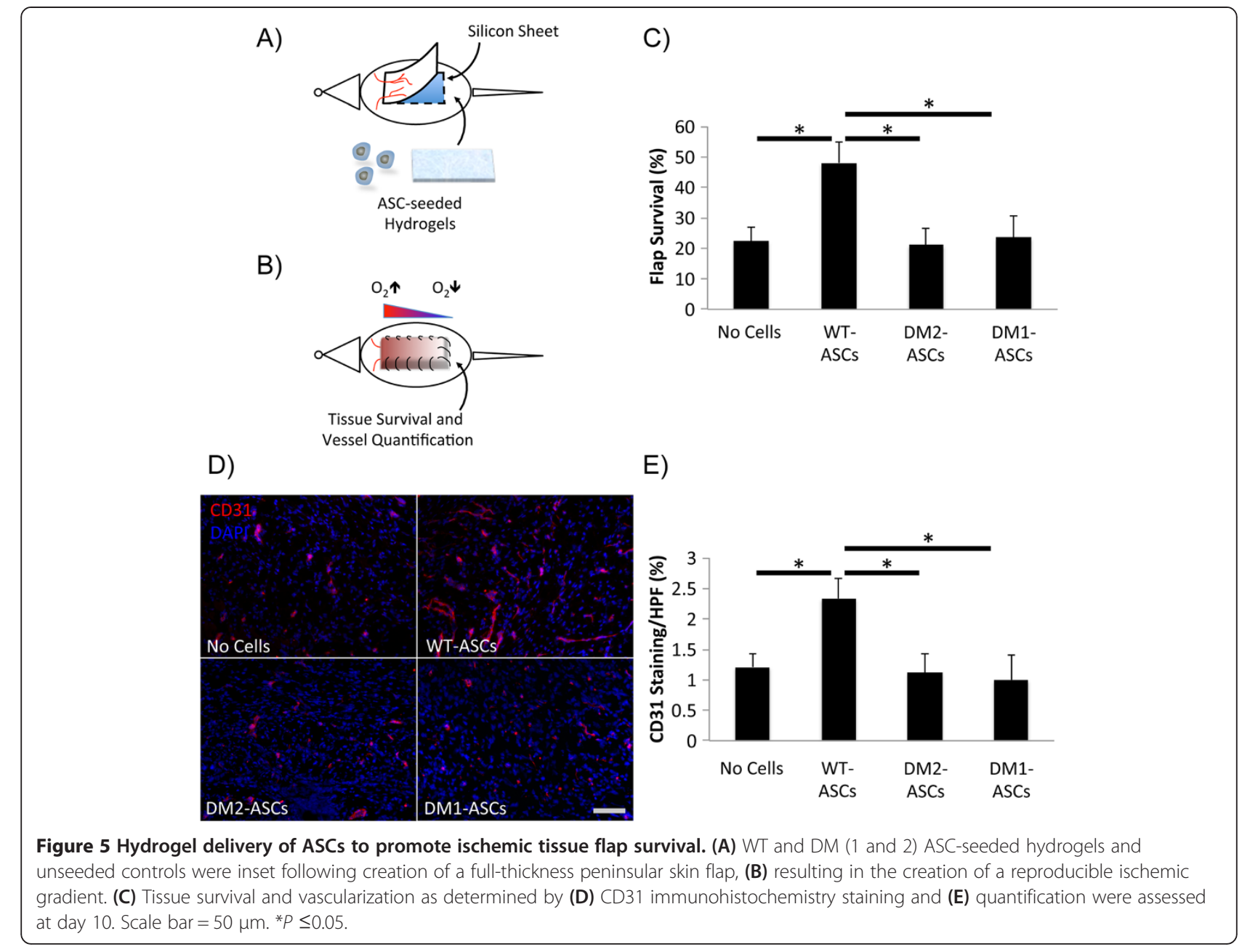

[18]. This analysis identified three distinct transcriptionally defined ASC subpopulations, which exhibited strikingly different profiles across conditions (Figure 7C-E). Interestingly, a distribution analysis of the cells comprising the third cluster revealed that there were considerably fewer diabetic ASCs compared with WT cells in this subpopulation (Figure $7 \mathrm{~F}$ ), with this cluster characterized in part by the elevated expression of genes associated with vasculogenesis, such as Angpt-1, Sdf-1, Mmp-3, and Fzd-4 (Figure 7G) [29,33-35]. These data suggest that this subpopulation may be primed to support vasculogenesis, and its depletion in the diabetic state provides a potential mechanism for the impairment of diabetic ASC vasculogenic potential.

\section{Discussion}

In this work, we provide a comprehensive analysis of the reduced therapeutic potential of diabetic ASCs for the promotion of tissue neovascularization and, for the first time, identify the selective depletion of a subpopulation of cells with a pro-vasculogenic phenotype in this setting. One of the main clinical advantages of ASCs is their ease of use in an autologous fashion. However, the dysfunctional signaling environment observed in diabetic fat pads suggests that prolonged hyperglycemia has multidimensional detrimental effects on ASC niche homoeostasis. Moreover, the continuous impairment of diabetic ASC functionality following isolation and culture within an optimized biomimetic hydrogel scaffold significantly limits the translational potential of autologously derived ASCs in this population.

Supporting the irreversibility of these diabetes-related impairments, the hydrogel scaffold used in this work was created specifically to recapitulate the stem cell niche and has been previously shown to enhance the pro-vasculogenic cytokine secretion (VEGF, MCP-1, FGF-1, MMPs, etc.) of bone marrow-derived mesenchymal stem cells (BM-MSCs), improve BM-MSC survival and engraftment within the high-oxidative-stress environment of ischemic murine skin wounds, and ultimately increase the angiogenic effect and wound-healing potential of BM-MSC-based therapies [7,8]. Similarly, when ASCs are seeded in this hydrogel, they display an upregulation of vasculogenesis-related genes as well as an 

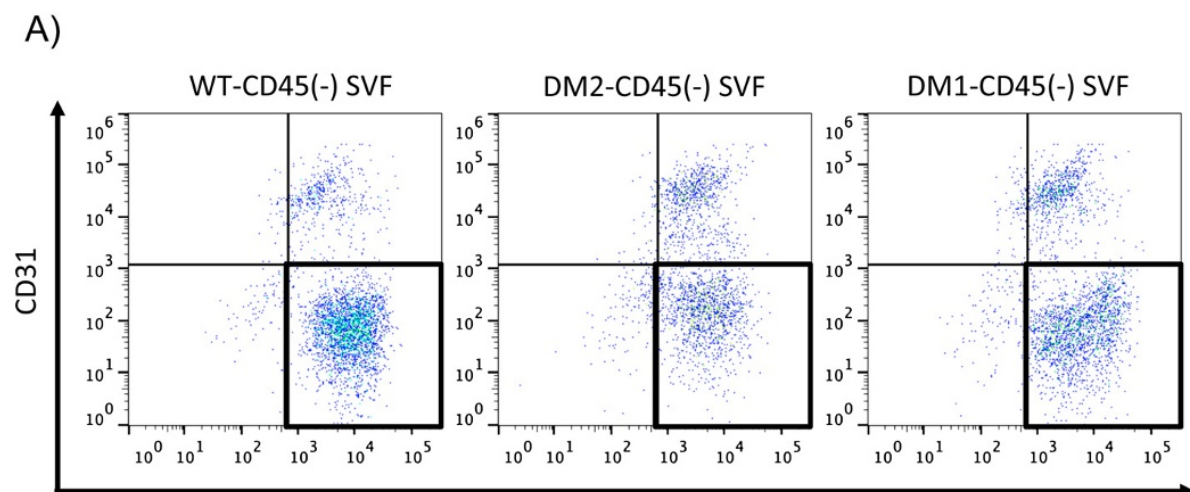

CD34

B)

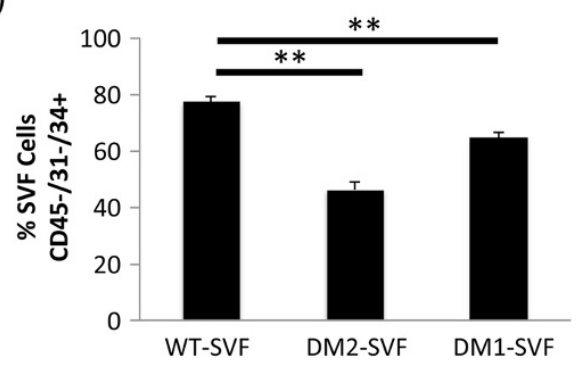

Figure 6 Diabetic and wild-type (WT) stromal vascular fraction (SVF) cell surface marker analysis. (A) Flow cytometric analysis determining the percentage of putative adipose-derived mesenchymal stem cells (ASCs) (CD $45^{-} / 31^{-} / 34^{+}$cells) within SVF obtained from diabetic (DM2 and DM1) and WT mice (CD45 and live/dead gating not shown). (B) Quantification of CD45 $/ 31^{-} / 34^{+}$ASCs in WT, DM2, and DM1 SVF reveals a significant depletion of ASCs in diabetic samples. ${ }^{* *} P \leq 0.01$

increased in vivo wound-healing potential (unpublished data). Given the proven efficacy of this hydrogel bioscaffold, the negligible beneficial effect of traditionally isolated diabetic ASCs when seeded in these constructs, particularly in promoting in vivo neovascular processes following ischemic injury, prompted us to explore the possibility of differential cell enrichment as a potential means to restore functionality.

Our recent work in the emerging field of microfluidicbased single-cell gene expression analysis supports the use of transcriptional-based cell clustering not only for the characterization of cell phenotypes [18] but also as a tool for functionality-based population enrichment [19]. Further justifying this approach, although others have shown that diabetes can negatively impact the therapeutic potential of the heterogeneous SVF for woundhealing applications [36], it remained unclear from this work whether this deficiency was the result of a global depletion of ASCs within the adipose tissue of diabetic animals or alternatively the manifestation of a changing membrane phenotype associated with impaired cell functionality.

Casting a wide net in our surface marker-based definition of ASCs, we observed a depletion of putative ASCs
$\left(\mathrm{CD} 45^{-} / \mathrm{CD} 31^{-} / \mathrm{CD} 34^{+}\right.$cells) within the diabetic SVF, which was consistent with the signaling dysfunction seen in this environment. The presence of a global ASC depletion does not exclude further phenotypic dysfunction, however, and the microfluidic-based approach allowed us to analyze the transcriptional profile of ASCs for phenotypic heterogeneity independent of a priori classifications. Thus, the depletion of a putatively vasculogenic ASC subpopulation in diabetes identified here not only provides further insight into the impaired phenotype of these cells but also suggests that the regenerative effects of healthy ASCs may be dependent upon a critical subpopulation of cells. Based upon these findings, work is currently ongoing to confirm the therapeutic efficacy of the identified subpopulation as well as to determine whether prospective enrichment of the depleted diabetic ASC subpopulation restores cell functionality.

\section{Conclusions}

The impaired therapeutic properties displayed by diabetic ASCs are likely related to the selective depletion of a highly vasculogenic subpopulation of cells. Although these data suggest that the utility of unaltered autologous ASCs for cell-based therapies in patients with 


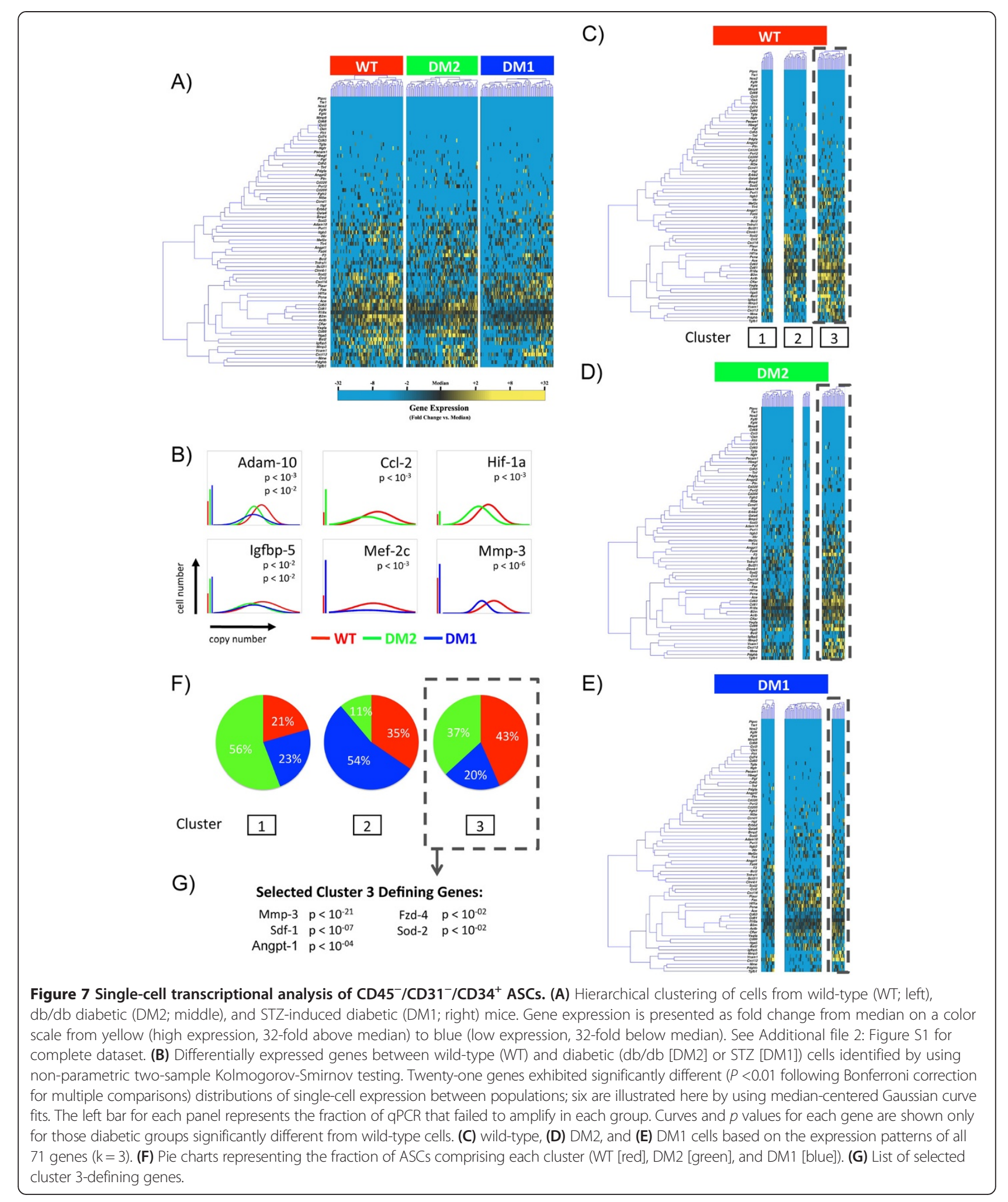


diabetes is limited, differential cell isolation techniques designed to enrich for depleted subpopulations are areas of future study that may potentially improve diabetic ASC function.

\section{Additional files}

Additional file 1: Table S1. Gene names and assay IDs for microfluidic single-cell gene expression analysis. Genes specifically relating to stemness and vasculogenesis were chosen, in addition to selected control, cell cycle, and surface marker-related probes.

Additional file 2: Figure S1. Whisker plots presenting raw quantitative polymerase chain reaction (qPCR) cycle threshold (Ct) values for each gene across all wild-type (WT) and diabetic adipose-derived mesenchymal stem cells. Individual dots represent single gene/cell qPCR reactions, and increased $\mathrm{Ct}$ values correspond to decreased mRNA content. Ct values of 40 were assigned to all reactions that failed to achieve detectable levels of amplification within $40 \mathrm{gPCR}$ cycles. Cells isolated from WT, db/db (DM2), and STZ (DM1) diabetic mice are colored in red, green, and blue, respectively. DM1, type 1 diabetes mellitus; DM2, type 2 diabetes mellitus.

\section{Abbreviations}

Angpt-1: angiopoietin 1; ASC: adipose-derived mesenchymal stem cell; DM: diabetes mellitus; DM1: type 1 diabetes mellitus; DM2: type 2 diabetes mellitus; DMEM: Dulbecco's modified Eagle's medium; FBS: fetal bovine serum; Fgf-2: fibroblast growth factor 2; Fgfr-2: fibroblast growth factor receptor 2; Hgf: hepatocyte growth factor; Hif-1a: hypoxia-inducible factor 1-alpha; MMP: matrix metalloproteinase; Pdgf-a: platelet-derived growth factor-A; qPCR: quantitative polymerase chain reaction; SVF: stromal vascular fraction; Vegf: vascular endothelial cell growth factor; WT: wild-type.

\section{Competing interests}

Funding for the stem cell research conducted in our laboratory has been provided by the Hagey Family Endowed Fund in Stem Cell Research and Regenerative Medicine, the Armed Forces Institute of Regenerative Medicine (US Department of Defense), the National Institutes of Health (R01-DK074095, R01-EB005718, and R01-AG025016), and the Oak Foundation. GCG is listed on the following patents assigned to Stanford University: (1) Intelligent Biodegradable Pullulan Regenerative Matrix for Tissue Engineering and (2) Efficient stem cell delivery into biomaterials using a novel capillary driven encapsulation technique. RCR and DD are also listed on patent (2). The other authors declare that they have no competing interests. Parts of this study were presented at the 7th Annual Academic Surgical Congress on 14-16 Feb. 2012.

\section{Authors' contributions}

RCR and MS contributed equally to the idea generation, experimental work, and manuscript preparation. MJ, DD, RK, MTC, JL, AR-D, SR, ZNM, MSH, JR, and MR contributed to the experimental work and manuscript preparation. GCG guided the idea generation, experimental work, and manuscript preparation. All authors contributed to the idea generation, design, and completion of this work and read and approved the final manuscript.

\section{Acknowledgments}

The authors thank Yujin Park for her assistance with tissue processing and staining. Flow cytometric analyses for this article were completed at the Stanford Shared FACS Facility. The authors thank the following researchers for their contributions to the data presented in this article: Michael Findlay, Wei Liu, and Toshihiro Fujiwara.

\section{Author details}

${ }^{1}$ Hagey Laboratory for Pediatric Regenerative Medicine; Department of Surgery, Stanford University School of Medicine, 257 Campus Drive West, Hagey Building GK-201, Stanford, CA 94305-5148, USA. ${ }^{2}$ Biomaterials and Advanced Drug Delivery Center, Stanford University, 1050 Arastradero Road, building A, Room A163 Palo Alto, Stanford, CA 94304, USA.
Received: 17 February 2014 Accepted: 12 June 2014

Published: 18 June 2014

\section{References}

1. Brem H, Tomic-Canic M: Cellular and molecular basis of wound healing in diabetes. J Clin Invest 2007, 117:1219-1222.

2. Tepper OM, Galiano RD, Capla JM, Kalka C, Gagne PJ, Jacobowitz GR, Levine JP, Gurtner GC: Human endothelial progenitor cells from type II diabetics exhibit impaired proliferation, adhesion, and incorporation into vascular structures. Circulation 2002, 106:2781-2786.

3. Lerman OZ, Galiano RD, Armour M, Levine JP, Gurtner GC: Cellular dysfunction in the diabetic fibroblast: impairment in migration, vascular endothelial growth factor production, and response to hypoxia. Am J Pathol 2003, 162:303-312.

4. Thangarajah H, Yao D, Chang El, Shi Y, Jazayeri L, Vial IN, Galiano RD, Du XL, Grogan R, Galvez MG, Januszyk M, Brownlee M, Gurtner GC: The molecular basis for impaired hypoxia-induced VEGF expression in diabetic tissues. Proc Natl Acad Sci U S A 2009, 106:13505-13510.

5. Thangarajah H, Vial IN, Grogan RH, Yao D, Shi Y, Januszyk M, Galiano RD, Chang El, Galvez MG, Glotzbach JP, Wong WW, Brownlee M, Gurtner GC: HIF-1alpha dysfunction in diabetes. Cell Cycle 2010, 9:75-79.

6. Rennert RC, Rodrigues M, Wong WW, Duscher D, Hu M, Maan Z, Sorkin M, Gurtner GC, Longaker MT: Biological therapies for the treatment of cutaneous wounds: phase III and launched therapies. Expert Opin Biol Ther 2013, 13:1523-1541.

7. Rustad KC, Wong WW, Sorkin M, Glotzbach JP, Major MR, Rajadas J, Longaker MT, Gurtner GC: Enhancement of mesenchymal stem cell angiogenic capacity and stemness by a biomimetic hydrogel scaffold. Biomaterials 2012, 33:80-90.

8. Wong W, Rustad KC, Glotzbach JP, Sorkin M, Inayathullah M, Major MR, Longaker MT, Rajadas J, Gurtner GC: Pullulan hydrogels improve mesenchymal stem cell delivery into high-oxidative-stress wounds. Macromol Biosci 2011, 11:1458-1466.

9. Wong W, Rustad KC, Galvez MG, Neofytou E, Glotzbach JP, Januszyk M, Major MR, Sorkin M, Longaker MT, Rajadas J, Gurtner GC: Engineered pullulan-collagen composite dermal hydrogels improve early cutaneous wound healing. Tissue Eng Part A 2011, 17:631-644.

10. Hong SJ, Traktuev DO, March KL: Therapeutic potential of adipose-derived stem cells in vascular growth and tissue repair. Curr Opin Organ Transplant 2010, 15:86-91.

11. Kim EK, Li G, Lee TJ, Hong JP: The effect of human adipose-derived stem cells on healing of ischemic wounds in a diabetic nude mouse model. Plast Reconstr Surg 2011, 128:387-394.

12. Maharlooei MK, Bagheri M, Solhjou Z, Jahromi BM, Akrami M, Rohani L, Monabati A, Noorafshan A, Omrani GR: Adipose tissue derived mesenchymal stem cell (AD-MSC) promotes skin wound healing in diabetic rats. Diabetes Res Clin Pract 2011, 93:228-234.

13. Rigotti G, Marchi A, Galie M, Baroni G, Benati D, Krampera M, Pasini A, Sbarbati A: Clinical treatment of radiotherapy tissue damage by lipoaspirate transplant: a healing process mediated by adipose-derived adult stem cells. Plast Reconstr Surg 2007, 119:1409-1422. Discussion 1423-1404.

14. Yan J, Tie G, Wang S, Messina KE, DiDato S, Guo S, Messina LM: Type 2 diabetes restricts multipotency of mesenchymal stem cells and impairs their capacity to augment postischemic neovascularization in $\mathrm{db} / \mathrm{db}$ mice. J Am Heart Assoc 2012, 1:e002238.

15. Jin $P$, Zhang $X$, Wu $Y$, Li L, Yin $Q$, Zheng $L$, Zhang $H$, Sun $C$ : Streptozotocin-induced diabetic rat-derived bone marrow mesenchymal stem cells have impaired abilities in proliferation, paracrine, antiapoptosis, and myogenic differentiation. Transplant Proc 2010, 42:2745-2752.

16. El-Ftesi S, Chang El, Longaker MT, Gurtner GC: Aging and diabetes impair the neovascular potential of adipose-derived stromal cells. Plast Reconstr Surg 2009, 123:475-485.

17. Gu JH, Lee JS, Kim DW, Yoon ES, Dhong ES: Neovascular potential of adipose-derived stromal cells (ASCs) from diabetic patients. Wound Repair Regen 2012, 20:243-252.

18. Glotzbach JP, Januszyk M, Vial IN, Wong WW, Gelbard A, Kalisky T, Thangarajah $H$ Longaker MT, Quake SR, Chu G, Gurtner GC: An information theoretic, microfluidic-based single-cell analysis permits identification of subpopulations among putatively homogeneous stem cells. PLoS One 2011, 6:e21211. 
19. Levi B, Wan DC, Glotzbach JP, Hyun J, Januszyk M, Montoro D, Sorkin M, James AW, Nelson ER, Li S, Quarto N, Lee M, Gurtner GC, Longaker MT: CD105 protein depletion enhances human adipose-derived stromal cell osteogenesis through reduction of transforming growth factor beta1 (TGF-beta1) signaling. J Biol Chem 2011, 286:39497-39509.

20. McEvoy RC, Andersson J, Sandler S, Hellerstrom C: Multiple low-dose streptozotocin-induced diabetes in the mouse. Evidence for stimulation of a cytotoxic cellular immune response against an insulin-producing beta cell line. J Clin Invest 1984, 74:715-722.

21. Albelda SM, Muller WA, Buck CA, Newman PJ: Molecular and cellular properties of PECAM-1 (endoCAM/CD31): a novel vascular cell-cell adhesion molecule. J Cell Biol 1991, 114:1059-1068.

22. Chung MT, Zimmermann AS, Paik KJ, Morrison SD, Hyun JS, Lo DD, McArdle A, Montoro DT, Walmsley GG, Senarath-Yapa K, Sorkin M, Rennert R, Chen $H H$, Chung AS, Vistnes D, Gurtner GC, Longaker MT, Wan DC: Isolation of human adipose-derived stromal cells using laser-assisted liposuction and their therapeutic potential in regenerative medicine. Stem Cells Transl Med 2013, 2:808-817.

23. Ceradini DJ, Kulkarni AR, Callaghan MJ, Tepper OM, Bastidas N, Kleinman ME, Capla JM, Galiano RD, Levine JP, Gurtner GC: Progenitor cell trafficking is regulated by hypoxic gradients through HIF-1 induction of SDF-1. Nat Med 2004, 10:858-864.

24. Suga H, Matsumoto D, Eto H, Inoue K, Aoi N, Kato H, Araki J, Yoshimura K: Functional implications of CD34 expression in human adipose-derived stem/progenitor cells. Stem Cells Dev 2009, 18:1201-1210.

25. Yang D, Wang W, Li L, Peng Y, Chen P, Huang H, Guo Y, Xia X, Wang Y, Wang $H$, Wang WE, Zeng C: The relative contribution of paracine effect versus direct differentiation on adipose-derived stem cell transplantation mediated cardiac repair. PLoS One 2013, 8:e59020.

26. Suga H, Rennert RC, Rodrigues M, Sorkin M, Glotzbach JP, Januszyk M, Fujiwara T, Longaker MT, Gurtner GC: Tracking the elusive fibrocyte: Identification and characterization of collagen producing hematopoietic lineage cells during murine wound healing. Stem Cells 2014, 32:1347-1360.

27. Wong WW, Sorkin M, Glotzbach JP, Longaker MT, Gurtner GC: Surgical approaches to create murine models of human wound healing. $J$ Biomed Biotechnol 2011, 2011:969618.

28. Potthoff MJ, Olson EN: MEF2: a central regulator of diverse developmental programs. Development 2007, 134:4131-4140.

29. Iohara K, Zheng L, Wake H, Ito M, Nabekura J, Wakita H, Nakamura H, Into T, Matsushita K, Nakashima M: A novel stem cell source for vasculogenesis in ischemia: subfraction of side population cells from dental pulp. Stem Cells 2008, 26:2408-2418.

30. van Hinsbergh WW, Koolwijk P: Endothelial sprouting and angiogenesis: matrix metalloproteinases in the lead. Cardiovasc Res 2008, 78:203-212.

31. Razban V, Lotfi AS, Soleimani M, Ahmadi H, Massumi M, Khajeh S, Ghaedi M, Arjmand S, Najavand S, Khoshdel A: HIF-1alpha overexpression Induces angiogenesis in Mesenchymal stem cells. Biores Open Access 2012, 1:174-183.

32. Stamatovic SM, Keep RF, Mostarica-Stojkovic M, Andjelkovic AV: CCL2 regulates angiogenesis via activation of Ets-1 transcription factor. J Immunol 2006, 177:2651-2661.

33. Babaei S, Teichert-Kuliszewska K, Zhang Q, Jones N, Dumont DJ, Stewart DJ: Angiogenic actions of angiopoietin-1 require endothelium-derived nitric oxide. Am J Pathol 2003, 162:1927-1936.

34. Deshane J, Chen S, Caballero S, Grochot-Przeczek A, Was H, Li Calzi S, Lach R, Hock TD, Chen B, Hill-Kapturczak N, Siegal GP, Dulak J, Jozkowicz A, Grant $M B$, Agarwal A: Stromal cell-derived factor 1 promotes angiogenesis via a heme oxygenase 1-dependent mechanism. J Exp Med 2007, 204:605-618.

35. Paes KT, Wang E, Henze K, Vogel P, Read R, Suwanichkul A, Kirkpatrick LL, Potter D, Newhouse MM, Rice DS: Frizzled 4 is required for retinal angiogenesis and maintenance of the blood-retina barrier. Invest Ophthalmol Vis Sci 2011, 52:6452-6461.

36. Cianfarani F, Toietta G, Di Rocco G, Cesareo E, Zambruno G, Odorisio T: Diabetes impairs adipose tissue-derived stem cell function and efficiency in promoting wound healing. Wound Repair Regen 2013, 21:545-553.

\section{doi:10.1186/scrt468}

Cite this article as: Rennert et al:: Diabetes impairs the angiogenic potential of adipose-derived stem cells by selectively depleting cellular subpopulations. Stem Cell Research \& Therapy 2014 5:79.

\section{Submit your next manuscript to BioMed Central and take full advantage of:}

- Convenient online submission

- Thorough peer review

- No space constraints or color figure charges

- Immediate publication on acceptance

- Inclusion in PubMed, CAS, Scopus and Google Scholar

- Research which is freely available for redistribution 